\title{
Conceptual design of hybrid photovoltaic-thermoelectric generator (PV/TEG) for automated greenhouse system
}

\begin{abstract}
Electrical energy is the highest set-up and operation cost in the agricultural greenhouse crop production in most of moderate or extreme temperature climate country. The energy consumes mostly for cooling, heating, ventilation, lighting, irrigation pumps, and the automation system. This paper proposed a theoretical approach and a conceptual design of a hybrid photovoltaic (PV) system by coupling PV panel and thermoelectric generator (TEG) modules which is to be applied in an Automated Greenhouse system project. The improved efficiency is to be compared between conventional PV generation system and by using various PV and TE module type configuration. Presented system is a conceptual design and further improvement will be considered such as combining with automated semi-transparent thin film solar panel to absorb excess radiation of sunlight during hot season. Simulation is also to be developed to validate the experimental approach.
\end{abstract}

Keyword: Solar; Photovoltaic (PV); Thermoelectricity; Thermoelectric generator (TEG); Hybrid system; Greenhouse system 